\title{
L'origine et la genèse de la conscience pure : Le contenu réel de Phénoménologie et matérialisme dialectique de Tran Duc Thao
}

\author{
Par JÉRÔME MELANÇON \\ University of Regina, Canada
}

Résumé La lecture de Husserl qu'effectue Tran Duc Thao vise à se débarrasser de la notion de conscience pure et ce, afin de retrouver la réalité de la vie humaine. Le marxisme permettra ainsi à Thao de dépasser la phénoménologie pour atteindre les buts propres à cette dernière. À partir de ce qu'il conserve de l'idéalisme transcendantal propre à Husserl, Thao développe un matérialisme dialectique où la subjectivité est le mouvement de la prise de conscience d'elle-même de la nature. Cet article propose une lecture de la manière dont Thao, dans Phénoménologie et matérialisme dialectique, retrace l'origine - dans l'histoire individuelle et collective - et la genèse dans l'intentionnalité propre aux philosophes - de la notion de conscience pure. Ce travail a lieu par un déplacement de la transcendance hors de l'idéalisme et dans le matérialisme; la fondation de l'expérience antéprédicative dans l'expérience matérielle; ainsi que l'examen de la formation de la conscience par le travail matériel et du poids des activités liées au travail dans la saisie d'elle-même que la conscience peut effectuer. Une fois écartée toute notion de pureté de la conscience, Thao peut retracer la genèse et l'origine réelles, matérielles, de la conscience dans les activités économiques et dans le langage.

Tran Duc Thao offre, dans Phénoménologie et matérialisme dialectique, une description de la création de la conscience pure par la philosophie, qui eut lieu par l'abandon du plan matériel, naturel de la réalité ; ainsi qu'une étude des activités de production, qui a permis l'émergence d'une conscience matérielle qui est occultée par la conscience pure, qui en recadre seulement 
les fonctions symboliques. Par le biais de son rapport à Husserl et de la créativité qu'il a mis à donner une nouvelle impulsion au matérialisme, Thao nous montre le chemin de ce qui pourrait être une phénoménologie matérialiste.

\section{Le problème du contenu de Phénoménologie et matérialisme dialec- tique}

En 1951, Tran Duc Thao rompt avec ses attaches en France, met fin au procès qui l'oppose à Jean-Paul Sartre et prépare son retour au Vietnam, sans doute grâce à l'accord et à l'aide du Viet Minh. Il publie aussi enfin les résultats des recherches philosophiques sur la phénoménologie d'Edmund Husserl qu'il mène depuis 1942, sous le titre Phénoménologie et matérialisme dialectique ${ }^{1}$. La publication de cet ouvrage pose deux problèmes d'ordre philosophique quant à la position qu'y défend Thao.

Tout d'abord, Thao avait rompu en 1948 avec la phénoménologie husserlienne "pour adhérer à la révolution que conduisent les communistes staliniens de son pays $»^{2}$. Il semble que c'est à partir de ce moment que son projet d'une étude dans la lignée de Husserl se transforme en une critique de la phénoménologie. Selon Thao, l'idéalisme transcendantal de Husserl aurait amené la philosophie ainsi que toute la tradition de pensée occidentale vers sa contradiction la plus aiguë. Les analyses que Husserl présente de l'expérience vécue révèlent à la fois l'impossibilité de penser l'existence humaine en termes idéalistes et le contenu matériel de toute expérience qui s'immisce même dans la philosophie la plus idéaliste. Sa critique porte avant tout sur le virage transcendantal de Husserl, qui mène à l'impossibilité de rendre compte de la réalité en évacuant sa matérialité. Néanmoins, Thao est en mesure d'offrir une reprise matérialiste de la phénoménologie husserlienne en se tournant vers ce qu'elle n'a pu s'empêcher de conserver et de décrire de la réalité matérielle, ne fût-ce qu'au passage.

\footnotetext{
${ }^{1}$ Nous citons ici l'édition de J. Benoist et M. Espagne (dans J. Benoist \& M. Espagne, dir., L'itinéraire de Tran Duc Thao. Phénoménologie et transferts culturels, suivi de Phénoménologie et matérialisme dialectique, Paris, Armand Colin, 2013) et, entre crochets, l'édition de 1951 (Paris, Minh-Tân), réimprimée en 1971 (Paris, Londres, New York, Gordon \& Breach).

${ }^{2}$ D. Hemery, «L'Homme, un itinéraire vietnamien. Humanisme et sujet humain au $\mathrm{XX}^{\mathrm{e}}$ siècle ", contribution au colloque " Humanisme vietnamien, les paradoxes de l'Histoire » du 25 mai 2002, Musée royal de Mariemont, Belgique. En ligne: http://www.europe-solidaire.org/spip.php?article5090
} 
Ensuite, le matérialisme dialectique que nous pouvons voir dans l'œuvre de 1951 - comme dans le reste de ses écrits français - est certes un marxisme, mais se situe fort loin de l'orthodoxie communiste de l'époque et de la pensée de Marx, même dans ses intentions. De longs passages de Marx y sont certes cités, mais il ne s'agit que des passages les plus fameux de la préface à la Contribution à la critique de l'économie politique, et seulement pour ajouter à l'explication que Thao donne de problèmes qui ne sont pas ceux de Marx. Ces citations mêmes ne sont pas commentées ni ne sont au cœur de la pensée de Thao — tout au plus marque-t-il par leur inclusion qu'il y trouve inspiration. Ainsi, les analyses que développe Thao s'appuient sur une théorie du reflet entre base et superstructure qui est tout autre que celle développée par les marxistes orthodoxes : elles visent à la description complète de l'intentionnalité, dans ses dimensions et comportementale et symbolique, telle qu'elle se crée dans l'histoire individuelle et sociale, et dans ses dimensions à la fois individuelle et sociale. Il s'agirait donc d'un matérialisme qui reprend les buts de la phénoménologie, plutôt que de mettre de l'avant l'exigence révolutionnaire.

À ces problèmes d'abord du rapport à Husserl et Marx et ensuite de l'unité du livre, nous offrons comme solution une lecture qui reprend le motif des études que Thao publia sur Hegel et Husserl: nous dévoilerons le « contenu réel » de Phénoménologie et matérialisme dialectique, à savoir une tentative de dépasser et l'idéalisme transcendantal et le marxisme vers une phénoménologie matérialiste. Ainsi, nous suggérons que ce livre peut être lu aujourd'hui non simplement en tant que document historique (comme Thao a pu le suggérer en $1992^{1}$ ), ni seulement en tant que tentative d'opérer une synthèse de Husserl et de Marx. Cette phénoménologie prend comme une mystification et un phénomène la conscience pure et tente de retracer et son origine historique dans le développement social et sa genèse dans le développement individuel, afin de retrouver en levant cette mystification la conscience réelle, à la fois individuelle et sociale, à la fois comportementale et symbolique.

Afin de montrer ce que Thao doit à Husserl et conserve de la phénoménologie tout en se défaisant de la théorie proprement husserlienne par des emprunts au marxisme qui le minent et jettent souterrainement les bases d'un tout autre matérialisme dialectique, nous développerons la critique explicite de la phénoménologie présentée par Thao, avant de montrer comment la matérialité se trouve dégagée par la phénoménologie et est son contenu réel.

1 Th. Marchaisse, «De quoi sommes-nous responsables ? », dans J. Benoist \& M. Espagne, dir., L'itinéraire de Tran Duc Thao, op. cit., p. 251-256. 
Nous pourrons ensuite expliquer la genèse de la conscience dans le mouvement du travail et de la production, pour enfin retracer les origines historiques de la conscience pure et les origines possibles d'une conscience bien comprise dans toute sa matérialité, en termes de co-participation et de communauté.

\section{La critique de la phénoménologie et le retour au matériel}

La critique de Tran Duc Thao à l'endroit de la phénoménologie husserlienne et du communisme français est déjà entamée dans un article contre l'interprétation de Hegel par Kojève, dont la philosophie fut importante pour la phénoménologie française même, au-delà des enjeux propres à l'interprétation de Hegel. Kojève, rappelons-le avec Thao, propose une lecture de la Phénoménologie de l'esprit comme entièrement ancrée dans la dialectique du maître et de l'esclave. Dans un échange de lettres avec Thao, Kojève avait d'ailleurs bien indiqué qu'il désirait par cette analyse de Hegel faire œuvre de propagande ${ }^{1}$. La critique de Kojève peut ainsi être vue comme celle d'un marxisme qui s'en remet à la dialectique du maître et de l'esclave.

Contre Kojève, Thao suggère que l'œuvre de Hegel doit être lue en termes matérialistes, et que la dialectique du maître et de l'esclave n'est pas la clé du livre, mais bien au contraire est elle-même un problème de possession et de propriété, marquant que le mouvement de la reconnaissance n'est pas établi entre le maître et l'esclave, mais bien au sein de la société dans toute son étendue - la reconnaissance est un phénomène social et non individuel. Kojève prend donc la mauvaise route marxiste, se concentrant sur la lutte des classes plutôt que sur le matérialisme. Pour Thao, la Phénoménologie hégélienne doit plutôt être lue comme trouvant son sens

${ }^{1}$ A. Kojève, «Document. Correspondance entre Alexandre Kojève et Tran-DucThao » (7 octobre 1948), dans G. Jarczyk \& P.-J. Labarrière, De Kojève à Hegel : cent cinquante ans de pensée hégélienne en France, Paris, Albin Michel, 1996, p. 64 : «mon œuvre n'avait pas le caractère d'une étude historique; il m'importait relativement peu de savoir ce que Hegel lui-même a voulu dire dans son livre; j'ai fait un cours d'anthropologie phénoménologique en me servant des textes hégéliens, mais en ne disant que ce que je considérais être la vérité, et en laissant tomber ce qui me semblait être, chez Hegel, une erreur. Ainsi, en renonçant au monisme hégélien, je me suis consciemment écarté de ce grand philosophe. D'autre part, mon cours était essentiellement une œuvre de propagande destinée à frapper les esprits. C'est pourquoi j'ai consciemment renforcé le rôle de la dialectique du Maître et de l'Esclave et, d'une manière générale, schématisé le contenu de la phénoménologie. » 
effectif dans le développement des forces productives, et donc dans la vie économique et matérielle, plutôt que politique. Cette lecture est possible parce que Hegel révèle malgré lui que le mouvement de la conscience est matériel et donc qu'à l'aboutissement de ce mouvement, « le capital est le pur intelligible» plutôt que ce soit la conscience qui forme le pur intelligible $^{1}$.

La Phénoménologie est donc limitée dans ses résultats, parce qu'elle s'en tient à « la partie consciente du mouvement, la seule qui se soit maintenue dans le souvenir de la subjectivité $»^{2}$. Hegel - comme la conscience philosophique, comme la conscience individuelle - oublie le travail réel qui donne au monde naturel, à l'être-là, un sens en soi, et effectue donc une distinction factice entre le pour-soi et l'en-soi. Une fois ce mouvement repris à partir de ce que Hegel en oublie, Thao peut montrer que les trois étapes majeures du développement de la conscience contiennent aussi le réel - c'est-à-dire le matériel.

Ainsi, la certitude sensible équivaut à la vie et à l'attitude naturelles ; la perception, à la logique de l'échange des marchandises et à l'influence de l'argent sur les rapports sociaux; et l'entendement, à la rationalité mathématique de la manufacture. Le pur intelligible qui correspond au mouvement de l'entendement est simplement le capital, qui ne se voit nulle part mais qui agit partout et qui fait que toute relation et tout être concret peut être calculé et à son tour transformé en capital. Ce contenu réel, Hegel ne le voit pas ; pour lui, ces problèmes matériels sont déjà résolus parce qu'il n'y a que le travail spirituel qui compte. C'est de la sorte que « le monde semble ainsi se créer par la réflexion du Soi sur soi-même ${ }^{3}$. La conscience ne se souvient que de ce sur quoi elle a réfléchi et en laisse de côté les assises matérielles, à savoir : la naturalité et l'animalité propres à toute corporéité ; le travail et les échanges monétaires qui séparent et aliènent l'individu des produits échangés par l'introduction d'une nouvelle médiation; et le travail des ouvriers exploités au stage où le capitaliste n'entretient en effet plus aucune relation directe — c'est-à-dire productive — au monde matériel, se plaçant à l'encontre des forces productives.

Thao développa la portée de ces idées dans la seconde partie de Phénoménologie et matérialisme dialectique. Il reprit également sa critique dirigée vers Hegel à l'endroit de Heidegger et Sartre dans la préface de ce

\footnotetext{
${ }^{1}$ Tran Duc Thao, «La Phénoménologie de l'esprit et son contenu réel », Les Temps Modernes, 36 (sept. 1948), p. 514.

${ }^{2}$ Ibid., p. 507.

${ }^{3}$ Ibid., p. 508.
} 
même livre, et à l'endroit de Husserl dans sa première partie. Ce qui nous intéresse ici est moins la rectitude de l'analyse que Thao présente de Husserl ${ }^{1}$ que ce à quoi sa lecture mène - à savoir le dégagement du «contenu réel » de ses textes, c'est-à-dire l'analyse du rapport de la conscience à la matérialité, analyse qui est chez Husserl à la fois présente au fil de ses autres analyses et empêchée dans son développement propre. La stratégie de lecture que Thao déploie le met ainsi à l'écart de ses contemporains. Comme nous l'avons vu, Kojève reprend l'un des mouvements majeurs de la pensée de Hegel et le redirige pour le mettre à jour, puisant chez lui librement pour bâtir autre chose, et mettant ses propres idées au compte de Hegel. Thao demeure davantage fidèle à Husserl et se montre fort soucieux de bien démontrer ce qui est prometteur dans sa pensée et doit être développé autrement. Il n'est bien entendu pas aussi fidèle à Husserl qu'a pu l'être à la même époque Jean-Toussaint Desanti à Marx, ou du moins au Marx de la doctrine du Parti Communiste Français (P.C.F.) ${ }^{2}$. Nous ne trouvons aucun souci d'orthodoxie, aucune obéissance à une autorité politique dans Phénoménologie et matérialisme dialectique — pas même celle, créative et idiosyncratique, qui animera Sartre dans ses tentatives de rapprochement du P.C.F. au début des années $1950^{3}$. En effet, Thao prend ses distances par rapport à Husserl en présentant une critique en bonne et due forme de celuici; et s'il nomme bel et bien Marx à quelques reprises, ses analyses matérialistes sont bel et bien les siennes propres, ouvrant une direction qu'il reprendra à nouveaux frais au moins à deux reprises - dans ses articles sur le langage et ses Recherches sur l'origine du langage et de la conscience et dans ses manuscrits du début des années $1990^{4}$. Thao se démarque enfin de

\footnotetext{
${ }^{1}$ Une telle analyse exigerait un tout autre travail de comparaison et de lecture plus rapprochée de Husserl; nous nous bornons donc ici à noter ce qui doit être connu de ses critiques pour comprendre ce que Thao conserve des analyses husserliennes.

${ }^{2}$ Le thème et les raisons de l'engagement des Desanti auprès du P.C.F. sont exposés au fil de D. Desanti \& J.-T. Desanti, avec R.-P. Droit, La liberté nous aime encore, Paris, Odile Jacob, 2004. Desanti développera plus tard sa propre philosophie en se rapprochant de Husserl pour s'éloigner de Marx. Voir notamment Le philosophe et les pouvoirs et autres dialogues, Paris, Hachette, 2008.

${ }^{3}$ Le texte qui en est le plus emblématique, «Les communistes et la paix » (Situations, VI, Paris, Gallimard, 1964, p. 80-253, et sa suite, « Réponse à Claude Lefort», Situations, VII, Paris, Gallimard, 1965, p. 7-93), fut l'une des pièces centrales menant à sa rupture avec Merleau-Ponty.

${ }^{4}$ Tran Duc Thao, Recherches sur l'origine du langage et de la conscience, Paris, Éditions sociales, 1973; de ses derniers travaux, mentionnons «La logique du présent vivant », Les Temps Modernes, 568, novembre 1993, p. 154-168, et Intro-
} 
l'approche de Merleau-Ponty, qui, comme ce dernier le lui avait confié, visait dans les années 1940 à faire une synthèse de Husserl, de Hegel et de Marx ${ }^{1}$. Cette synthèse (avec l'apport de la psychologie de la forme) était néanmoins originale, chaque philosophe servant à Merleau-Ponty à surmonter les problèmes propres aux autres, le tout en vue de ses propres questionnements et de ses propres analyses de la perception ainsi que de la vie politique de l'époque. Tandis que Merleau-Ponty pouvait s'inscrire dans la mouvance phénoménologique tout en se distinguant de Husserl, Thao, au contraire, choisit la mouvance du matérialisme dialectique comme ancrage. À partir de ces choix chacun se dirige dans une direction qui lui est propre et qui fera l'objet de tentatives renouvelées, autour du même problème : l'ancrage du sens dans la nature.

Thao définit le matérialisme dialectique et le développe par le biais d'une étude de textes provenant de diverses disciplines et offrant des descriptions de la vie matérielle, le tout en s'inspirant de Marx, sans le commenter ou l'interpréter, sans s'appuyer sur la doctrine marxiste ou sur l'orthodoxie communiste. Le matérialisme dialectique, qu'il ne nomme pas « marxisme », n'est donc pas avant tout pour lui la pensée de Marx. Thao fonctionne autrement dans la première partie de son livre, qui consiste en une étude des textes, et en grande partie des inédits, de Husserl. Il ressort de ces relations aux textes que pour Thao, la phénoménologie équivaut sans doute à la pensée de Husserl. Il présente celle-ci comme une pensée en évolution, émergeant d'une réflexion sur les mathématiques pour se développer jusqu'à des descriptions de vécus intersubjectifs dans ses inédits tardifs, renouvelée à plusieurs reprises pour faire face à ses problèmes. Thao marque bien aussi que cette pensée, ancrée dans la tradition philosophique classique, est reprise et continuée par l'existentialisme qui la renouvelle d'une nouvelle manière, mais encore une fois dans la mauvaise direction. Il retire de l'œuvre de Husserl une compréhension de la phénoménologie comme une recherche de l'invariant par le biais de ce qui est impossible à concevoir ou imaginer et qui montrera ainsi les conditions de possibilité de la réalité étudiée - c'est-àdire l'essence ${ }^{2}$. Il explique par ailleurs le but de l'analyse phénoménologique

duction au problème de l'homme - La liaison du biologique, du social et du psychique (manuscrit édité par l'auteur, février 1992).

${ }^{1}$ Tran Duc Thao, « Recherches dialectiques. (I) Un itinéraire » (manuscrit édité par l'auteur puis annoté, avril 1992), p. 20. Pour les textes de Merleau-Ponty qui se rapprochent le plus de cette tentative, voir surtout Sens et non-sens, Paris, Gallimard, 1996.

${ }^{2}$ Tran Duc Thao, Phénoménologie et matérialisme dialectique, op. cit., p. 280-286. 
comme étant la recherche des conditions de l'évidence, des conditions du sens, afin d'en arriver à décrire le sens d'un objet, de l'être, du moi, et même de chaque vécu ${ }^{1}$. Il cherchera de la sorte à conserver ce que Husserl fait de mieux, c'est-à-dire expliciter le sens du donné vécu, tout en tentant d'accomplir ce qu'à son avis Husserl visait sans réussir à le faire, à savoir demeurer sur le plan du concret ${ }^{2}$.

Thao rappelle que Husserl développa la théorie phénoménologique sous trois formes distinctes, à chaque fois recommençant la même tentative d'en revenir aux choses mêmes, autrement dit au réel, pour surmonter la distance entre la théorie et sa pratique qui se creusait à chacune de ses tentatives : la description des essences, l'explicitation du vécu, et l'explicitation génétique. Cet écart entre la théorie et la pratique répète celui que Thao trouvait chez Hegel. L'idéalisme, peu importe sa forme, est une philosophie négative qui ne prend pour objet que le contenu explicite, réflexif de la pensée, même lorsqu'elle cherche l'antéprédicatif :

L'horizon même de la phénoménologie détourne le regard du phénoménologue des données réelles qui définissent pourtant le contenu véritable de sa réflexion. Plus précisément elles ne lui apparaissent que sous leur aspect négatif, en tant qu'elles se suppriment dans leur signification. Or, le mouvement effectif de la description renvoie inéluctablement à cette réalité matérielle, qui s'y révèle constamment comme la couche ultime des formations constituées ${ }^{3}$.

La contradiction de la phénoménologie est qu'elle revient sans cesse, malgré elle, à la réalité matérielle, sans pour autant être à même de la décrire effectivement.

La phénoménologie est par ailleurs impuissante à définir et circonscrire la matérialité, puisqu'elle en ignore le ressort, à savoir les objets du monde et le rapport qu'entretient le sujet avec eux, qui n'est pas un rapport de sensation, de perception, ou de signification, mais bien un rapport de

\footnotetext{
${ }^{1}$ Ibid., p. 291, 295, 297.

${ }^{2}$ Ibid., p. 303, 297. Notons cependant que Thao démontre que Husserl transforme la phénoménologie en cherchant le chemin qui le ramènera à la réalité matérielle, et non pas que ce chemin (1) n'a pas été retrouvé ni (2) est impossible à retrouver. Nous pourrions donc voir chez lui le choix d'un autre chemin que ceux tracés par Husserl, celui du matérialisme dialectique, comme plus approprié à cette recherche des conditions de possibilité de la relation à la réalité matérielle. Nous remercions le lecteur anonyme du Bulletin d'avoir fourni l'impulsion à cette précision : mettre la réalité matérielle entre parenthèses n'est pas la nier.
}

${ }^{3}$ Ibid., p. 270 [10]. 
production. Pour Thao, est matériel tout ce qui est engagé dans un rapport de production; ce qui en est abstrait n'en est pas moins matériel, mais la conscience qui effectue cette abstraction est en contradiction avec la réalité matérielle en ce qu'elle se cache ses propres liens avec elle pour ne se faire que sens, connaissance et expérience. Thao reprend néanmoins la relation étroite que maintient la phénoménologie entre le sujet et l'objet, la conscience et le monde, l'un ne pouvant être compris sans l'autre. Ainsi, l'« être effectivement réel » est «le travail réel des classes opprimées », "la réalité effective du travail producteur », «la matérialité créatrice ${ }^{1}-$ autrement dit, la réalité est le travail de production en contact avec le matériel, c'est-à-dire la matière qui peut servir à ce travail. L'être humain est également matériel, homogène à la matière : il travaille, il peut être utilisé pour le travail, et se trouve ainsi dans un rapport matériel de production, un rapport au sein duquel émerge le sens. La conscience n'ajoute donc pas des significations à la matérialité ; elle les y trouve. La matérialité, dans la conscience même,

définit la couche originaire dont le mouvement engendre les modes plus élevés de l'être, dans la spécificité de leur sens, l'infrastructure réelle qui fonde les superstructures idéales dans leur surgissement historique et dans leur valeur de vérité ${ }^{2}$.

La matérialité se trouve également dans des « rapports sociaux de production qui définissent le contenu réel de la vie sensible ${ }^{3}:$ la vie de la conscience est à la fois matérielle et sociale ; par la production, la conscience est en rapport constant à la réalité matérielle et développe au sein de ce rapport une réalité symbolique à laquelle la phénoménologie se limite ${ }^{4}$. La transcendance, que rejette Thao, ne peut avoir lieu qu'une fois que les intentions, les motifs, les données sensibles et les formations sensori-motrices sont détachées du rapport de production à la réalité matérielle, détachées donc du premier rapport créateur qui cherche à penser le matériel dans le mouvement

\footnotetext{
${ }^{1}$ Ibid., p. $272-273$

${ }^{2}$ Ibid., p. 269.

${ }^{3}$ Ibid., p. 273 .

${ }^{4}$ Orientés de manière similaire, Merleau-Ponty, dans ses travaux sur la pédagogie, sur l'institution, puis sur la nature, s'attache à décrire cette fonction symbolique, tandis que Claude Lefort et Cornelius Castoriadis s'efforcent de développer cette dimension symbolique en relation à la vie sociale et politique. Voir notamment C. Castoriadis, L'institution imaginaire de la société, Paris, Seuil, 1975, et C. Lefort, Les formes de l'histoire. Essais d'anthropologie politique, Paris, Gallimard, 1978.
} 
même de sa transformation. Nous verrons d'ailleurs comment Thao décrit l'émergence de la conscience à partir de cette vie matérielle et sociale et de ces rapports de production.

Cette contradiction entre théorie et pratique - entre la conscience pure, épurée, et la conscience matérielle qui la sous-tend - est la source de la tentation existentialiste telle qu'elle est développée par Heidegger: puisque le monde est en la conscience et qu'elle se perçoit en lui, être-dansle-monde est une structure ontologique de la conscience. C'est d'être homme qui fait qu'elle peut être dans le monde, monde qu'elle porte en elle comme son projet. L'absence de justification et l'arbitraire du projet sont les seuls fondements du monde. Par l'œuvre de Heidegger,

toutes les valeurs acquises par le long travail de la tradition humaniste se découvrent subitement comme dénuées de fondement réel, et ne se maintiennent plus qu'en s'accrochant à la «décision-résolue » de défendre jusqu'à la mort ce qu'on est devenu incapable de justifier en raison et en vérité $^{1}$.

C'est non seulement Heidegger, mais aussi Sartre que Thao vise ici : l'idée que l'existence précède l'essence devient le postulat que l'idée précède la matérialité, que la pensée contient en elle toute la réalité, que la subjectivité est à même de choisir le monde.

Cette liquidation rapide de l'existentialisme effectuée, Thao peut se concentrer sur son propos, qui ramène les problèmes de l'existentialisme à une hypertrophie des problèmes propres à la phénoménologie husserlienne : si la pratique phénoménologique échoue dans sa tentative de retourner aux choses mêmes — donc de retourner à la matérialité - c'est du fait des manquements de la théorie. Le problème de la pensée husserlienne ne se décèle toutefois pas du côté de sa théorie, mais se révèle de celui de la pratique, de ses résultats - surtout dans les inédits. Ces documents montrent l'incompatibilité des analyses concrètes et des principes théoriques, une difficulté à laquelle ne peut remédier l'aboutissement des analyses concrètes ou même un élargissement de la perspective phénoménologique. Au contraire, pour Thao «la seule énumération des analyses concrètes [...] entrâ̂nait une rupture totale et définitive avec les principes fondamentaux de la doctrine $»^{2}$.

\footnotetext{
${ }^{1}$ Ibid., p. 271 [12].

${ }^{2}$ Ibid., p. 268 [6]. Thao, sauf dans son explication de ce que fait la phénoménologie (voir plus haut), ne présente pas ce que seraient ces « principes fondamentaux ».
} 
La phénoménologie, affirme Thao, est contredite par l'absorption du point de vue de la conscience dans la réalité naturelle ; c'est en reprenant ce mouvement dialectique que Thao tente de dépasser la phénoménologie. Le monde sensible se définit en effet par les structures du comportement et non par le pur vécu et « la description des significations antéprédicatives renvoie aux conditions de l'existence matérielle et place nécessairement le sujet dans le cadre de la réalité objective $»{ }^{1}$. Par ailleurs, la vie sensible passe par la production des conditions de l'existence humaine, et ce sont ses formes techniques et économiques qui sont à décrire - ce qu'indique déjà Husserl dans L'Origine de la géométrie ${ }^{2}$ par sa tentative de fonder la vérité sur la praxis.

Si Husserl cherchait à revenir à la conscience transcendantale, c'était du fait de l'insuffisance des sciences; or, il aboutit à une philosophie qui est selon Thao tout aussi insuffisante, totalement sceptique, à un relativisme du savoir où toutes les vérités se valent, et où l'imagination doit même être préférée à l'entendement (comme lorsqu'il affirme que la Terre n'est pas un corps céleste parmi les autres). Thao renvoie l'échec du retour aux choses à une erreur du projet initial de Husserl : s'il s'agissait de revenir à « la chose même ", cette chose devait être l'homme réel, chose qui produit du sens par son travail sur les autres choses. Dissiper le monde des apparences propre à la conscience perceptive exigeait que Husserl le mette entre parenthèses, nous ramenant à un monde que nous constituons dans un mouvement de retrait que redouble l'épochè. Le monde réel devenait le monde irréel appartenant à une conscience pure : réel, mais uniquement parce que constitué; connu, mais seulement parce que découvert au sein de la réduction, après que la question de son existence ait été suspendue sans jamais être résolue, hors de toute relation à la matérialité et à la corporéité propre à l'être humain.

Or, purifier la conscience de ses rapports à la matérialité est exactement la mystification que repère le matérialisme dialectique dans la pensée bourgeoise : la transcendance s'avère être le remplacement des significations qui émergent du travail producteur - travail de la conscience matérielle et productive - par les significations produites à partir des positions privilégiées, expropriatrices et exploiteuses, afin de nier la réalité du travail effectué par les autres consciences. L'arrivée du prolétariat sur la scène historique présente par conséquent une transformation radicale du rapport à la réalité en même temps qu'une possibilité de révolution. Tandis que l'idéologie suggère aux producteurs qu'ils peuvent espérer améliorer leur sort et reprendre le

${ }^{1}$ Ibid., p. 404 [219].

${ }^{2}$ E. Husserl, L'Origine de la géométrie, Paris, PUF, 2010. 
contrôle de la production en passant à leur tour à la position d'exploiteurs, les rapports de force et le manque de ressources matérielles empêchent un tel espoir d'échapper au travail productif et exploiteur de l'intérieur même des institutions économiques et politiques en place. Nombre d'anciens capitalistes se joignent par ailleurs à leurs rangs à mesure que la logique de l'exploitation s'étend à eux, les forçant ainsi à vivre le travail productif plutôt que de s'en faire une idée abstraite et quantifiable ${ }^{1}$. Les producteurs ne peuvent par conséquent voir l'idéalisme, que développent des consciences sans lien au travail et dans un rapport aux autres consciences qui n'est qu'individuel, que comme un masque pour le "matérialisme sordide ${ }^{2}$ qu'est la pratique bourgeoise d'exploitation. Les producteurs ressentent ainsi le besoin de défendre la matérialité de leur production et les valeurs qui l'accompagnent. Thao esquisse de la sorte ce qui relie les manquements de la philosophie aux manquements de la société et de l'ordre économique où elle a lieu. La tâche de la révolution prolétarienne est alors de prendre en charge les intérêts de l'humanité. Cette révolution émerge de la vie matérielle, par la dialectique qui lui est propre. On voit ainsi à nouveau « que le matérialisme est l'origine authentique de toute signification et de toute valeur $»^{3}$.

Une fois cette critique déployée, il ne demeure de la phénoménologie que ce qu'elle partage avec le matérialisme dialectique, à savoir la conscience matérielle, la phénoménologie de la chose et de la nature matérielle - éléments philosophiques qui sont la base d'une phénoménologie nonhusserlienne chez Thao. Le matérialisme dialectique, suggère-t-il, a trouvé depuis longtemps les réalités matérielles comme fondement de la constitution des significations et à laquelle vient tout juste d'arriver la phénoménologie : la production, dans les produits humains, crée le sens, qui en est la transposition symbolique, la reproduction, dans la conscience, et dont la vérité dépend du mode de production. La phénoménologie eut certes le mérite de liquider le formalisme et de ramener les valeurs au concret, mais elle ne peut évoluer sur ce terrain, elle ne peut procéder à la description des déterminations matérielles. Il s'agit donc de la réaliser en la supprimant en tant qu'idéalisme transcendantal, mais en empruntant le chemin qu'elle a ouvert

\footnotetext{
${ }^{1}$ Thao fait ici référence à la théorie du déclassement, comprise comme la «prolétarianisation » de la classe capitaliste, dont les membres se voient forcés à travailler au compte d'autrui à mesure que la compétition et la concentration du capital les ruinent.
}

${ }^{2}$ Ibid., p. 275 [17].

${ }^{3}$ Ibid., p. 275 [18]. 
vers le réel : il ne s'agit pas de reprendre simplement le chemin tracé par le matérialisme dialectique du passé.

La phénoménologie, par ses descriptions du vécu, a trouvé le fondement de tout sens - le sensible - , mais a perdu de vue le contenu matériel de la vie sensible, tomant dans la contingence absolue et le relativisme sensualiste. Thao prend ainsi les choses et la matérialité comme points de départ :

Dans le processus réel de production, l'homme est homogène à la matière et c'est dans ce rapport matériel lui-même que se constitue le rapport originaire de la conscience à l'objet qu'elle vise, comme « sens constitué »" .

Loin d'être une identité séparée de la réalité matérielle dont le monde n'est qu'une qualification parmi tant d'autres, la subjectivité est un mouvement qui enveloppe les opposés dialectiquement au sein de l'histoire, elle est la nature en tant qu'elle devient sujet - et la forme de ce mouvement est la matérialité. Le point d'arrivée est donc le matérialisme dialectique, qui est la vérité de l'idéalisme transcendantal, et la subjectivité constituante est le mouvement de la prise de conscience d'elle-même de la nature.

\section{Matérialité et idéalisme}

Il reste donc à décrire les rapports de la matière à la conscience - ce qui est l'objet du matérialisme dialectique, de la seconde partie de l'ouvrage de Tran Duc Thao, ainsi que de ses recherches subséquentes. Pour Thao en effet, le sort de la phénoménologie se joue dans deux passages qu'Husserl tente d'effectuer à partir du présent vivant que la réduction transcendantale découvre : d'abord, à l'apparition de l'intentionnalité première, à une description qui se limite aux pures impressions, sans constituer leur unité, à savoir, «le flot originaire du Présent absolu $»^{2}$; puis, de là, à la prédication et à la constitution.

Le présent vivant dont la conscience transcendantale fait l'expérience est une série d'impressions qui passent et se succèdent les unes aux autres. La conscience est donc passive, elle est le lieu où le présent vivant se déroule, elle est le présent vivant, et elle est donc aussi le présent vivant qui se prend pour objet tel qu'il vient tout juste d'être, se maintient par la

${ }^{1}$ Ibid., p. $274[16]$.

${ }^{2}$ Ibid., p. 401 [215]. 
rétention, et se continue ainsi lui-même. Ce glissement entre le présent et le passé qui fait leur unité est la sensation; parmi les sensations, les kinesthèses, qui organisent le sensible dans un champ sensoriel en vue de mouvements, permettent aussi un glissement du présent vers l'avenir. De telles intentionnalités sont passives, elles ne sont que des réactions à ce qui est senti. Dans le passage des kinesthèses, qui esquissent les mouvements possibles, aux protensions, où le mouvement s'accomplit et où l'intentionnalité se fait active, le sens interne du sensible se constitue. Un choix peut être accompli.

Les kinesthèses et protensions font apparaître l'extériorité au sein du champ sensoriel - à l'intérieur même de la conscience - sous la forme de configurations du champ sensoriel, de perspectives. Les kinesthèses orientent la conscience vers les choses qui apparaissent comme des objets-fantômes, qui malgré leurs déplacements et ceux de la conscience maintiennent leur unité par leur style, c'est-à-dire leur manière de se présenter et de se mouvoir, leur régularité, qui permet à la conscience de s'en emparer et de former des habitudes. La réalité sensible se constitue dans la dialectique entre le style et l'habitude.

$\mathrm{Au}$ sein de cette réalité sensible, la conscience transcendantale a l'expérience de son propre corps comme d'une telle chose-fantôme, chose vivante que la conscience anime, qui a ses résistances et donc son propre style et qui se porte de la sorte à des habitudes - mais elle demeure de ce même fait en extériorité à la conscience, de la même manière que les autres organismes vivants sont analogues au sien. L'autre corps vivant est comme le sien, comme il serait s'il était là-bas, où se trouve cette chose qui se comporte comme elle s'y comporterait, ce que Thao résume avec Husserl : «l'autre que je vois, n'est précisément que moi-même là-bas »" ${ }^{1}$. De là, des significations sociales peuvent s'ajouter aux significations internes, et la prédication est possible, puisqu'une autre conscience se devine dans le style de cet autre corps, avec les mêmes capacités de parler.

De la sorte, Thao montre comment dans la phénoménologie husserlienne, tout nouveau sens se trouve à l'aboutissement d'une chaîne de transferts de sens qui commence avec le sensible. Comme le décrit Husserl ${ }^{2}$, nous connaissons un objet suivant son type, c'est-à-dire suivant notre expérience des objets du même type faite par le passé. L'objet nouveau est saisi et dans sa nouveauté, et dans sa relation à un sens pré-existant, donc par

\footnotetext{
${ }^{1}$ Ibid., p. 403 [217].

${ }^{2}$ E. Husserl, Méditations cartésiennes et les Conférences de Paris, Paris, PUF, 1994, $\S 50$.
} 
transposition analogique d'un sens. Cette création de sens (Stiftung) est toujours liée à une création originaire (Urstiftung) dont elle est le transfert analogique et qui est son origine; elle est aussi liée à une genèse du sens dans le sensible et dans les configurations du champ sensoriel esquissées par les kinesthèses et les protensions.

En cela, Thao donne raison à Husserl ; il reprendra d'ailleurs ce que nous nommerons la dialectique de l'origine et de la genèse, où le sens dépend à la fois du sens créé d'avance, du fait de nos expériences passées et de nos contacts sociaux, et du fait de l'intentionnalité. L'origine peut être conçue comme un phénomène historique et social et la genèse, comme un phénomène géographique et personnel. Toute création de sens repose sur des créations de sens préalables, et aucune ne surgit de nulle part: il y a une chaîne historique du sens et de la praxis chez les autres qui appelle d'autres créations de sens et d'autres comportements et actions chez soi. Nous pouvons faire la chronologie de cette création du sens et cette praxis qui reprennent celles accomplies par d'autres — ou par soi — dans le passé. De même, toute création de sens repose sur un champ sensoriel que les kinesthèses et les protensions esquissent, et en émerge donc: il y a un enracinement géographique, lié à ce qui a lieu dans le corps à un endroit précis et lié à l'intentionnalité personnelle. Nous pouvons faire la généalogie de cette création de sens et de cette praxis qui répondent à un environnement ou milieu particulier.

Toutefois, Thao oppose à Husserl la contradiction fondamentale de la phénoménologie: la phénoménologie désirait suspendre la praxis et le monde de la vie. Ils refont surface dans ses analyses comme ce qui est déjà acquis par la conscience constituante, avant même de constituer quoi que ce soit. Le problème est que la vie matérielle n'apparaît que comme vie vécue et que le sol originaire de toute constitution et même de toute subjectivité est alors l'expérience comprise comme rajoutée à la conscience, tandis que la création originaire a en fait lieu dans la vie matérielle, dans la praxis comme rapport à la matérialité. C'est d'ailleurs selon Thao ce que Husserl reconnaissait dans l'explication génétique au cœur de L'Origine de la géométrie, sans pour autant arriver à retourner à la vie matérielle.

D'où la contradiction que relève Thao : ou bien Husserl ne revient jamais au monde qui fut mis entre parenthèses (donc la phénoménologie échoue), ou bien il n'en est jamais sorti (donc la phénoménologie telle que l'entend Husserl n'a jamais eu lieu). Autrement dit, ou bien il ne revient pas des significations aux choses mêmes; ou bien les significations sont les choses mêmes et la réduction transcendantale n'a jamais eu lieu. Thao refuse la réduction au transcendantal, à la pure vie de l'ego, suite à laquelle le 
phénoménologue ne doit plus voir que le sens de la manière dont le monde se présente à lui et dont il lui donne sens ${ }^{1}$. Ce refus de Thao est ancré dans sa conception de la donation du monde et du sens comme ayant lieu dans le travail de production même et non pas en surplus à celui-ci. Au contraire, le résultat de la réduction est que le sens d'être des objets les catégorise comme choses matérielles, organismes vivants, ou existants humains, mais seulement comme l'un de ces objets : un organisme vivant cesse d'être une chose matérielle; un existant humain cesse d'être vivant et matériel, parce que l'humanité est comprise comme la négation de la vie et de la matérialité. Ces choses matérielles sont par ailleurs transformées en objets culturels par la production humaine, c'est-à-dire par leur usage et leur manipulation, mais de telle sorte à nier leur matérialité pour se concentrer sur leur idéalité — sur leur sens culturel, alors que ces objets cessent d'être compris comme matériels. Le sens est toujours présupposé et préexiste donc toujours au mouvement; «l'homme ne s'explique que par l'homme » ${ }^{2}$ pour Husserl, comme pour Aristote et pour la tradition métaphysique, qui laissent de côté le matériel qui le dépasse et qui est le domaine d'émergence du sens.

Or, cette contradiction n'est présente que pour la phénoménologie qui a voulu abandonner le monde de l'existence empirique. L'erreur de Husserl a été de confondre l'attitude naturelle et l'attitude naïve : les circonstances empiriques sont la réalité naturelle, et ne peuvent être saisies par la conscience naïve. La conscience naïve prend les significations comme des réalités ; de ce fait, la réalité matérielle devient vide, ne prenant son sens que des actes qui la transcendent et qui s'y rajoutent. En refusant de la séparer de l'attitude naturelle, Husserl reprend en pratique l'attitude naïve et rejette la conscience naturelle qui saisit son travail productif comme source des significations. La supposition à l'origine de cette séparation entre le moi et le monde est que l'être n'a aucune signification par lui-même - supposition centrale à la conscience naïve, mais non à la conscience naturelle. Contre Husserl, Thao opère un rapprochement entre le sens et l'être. Les différents modes de l'existence sont des abstractions, des catégories, qui une fois substantifiées séparent le sens de chaque domaine d'être de l'être même. Ces catégories trouvent leur source dans ces activités de l'être humain par lesquelles il se donne sens à lui-même comme pure conscience constituante, sans relation à l'être - activités que Husserl privilégie exclusivement.

Malgré ces opérations, la phénoménologie nous montre bel et bien que la conscience constituante ne peut constituer qu'à partir du sens déjà

${ }^{1}$ E. Husserl, Méditations cartésiennes, op. cit., p. 65-66.

${ }^{2}$ Ibid., p. 408 [226]. 
constitué, qu'à partir de l'Urstiftung, qu'elle est ainsi un mouvement ancré dans la réalité, historique comme elle, ajoutant du sens au sens ancré dans l'être. Une fois la conscience replacée de la sorte au sein d'une réalité qui la dépasse et sur laquelle elle s'appuie, même dans ce qu'elle fait de plus abstrait, la réalité sera vue comme le devenir concret de l'être, dont les domaines sont des abstractions sur ce fond de concrétude. "Plus précisément, ajoute-t-il, elle [la réalité] est la nature elle-même dans son devenirsujet $^{1}$. " C'est la nature, autrement dit la matérialité, auxquelles participe la conscience, qui est l'originaire, l'Urstiftung — origine de tout mouvement, de toute pensée, de toute conscience - et la conscience est présente dans la nature par sa matérialité, c'est-à-dire par son corps, sa vie, sa conduite et ses activités.

En effet, lorsque Husserl décrit le processus d'unification de la chose par les kinesthèses et protensions, il ne parle de rien d'autre que de la conduite en relation à cette chose. L'instrumentalité du bâton, par exemple, et donc sa signification antéprédicative, est déterminée par l'allongement du bras qui prend prise puis s'en sert, et cette instrumentalité définit ensuite la réflexion, c'est-à-dire la signification prédicative de l'objet qui se solidifie dans la rétention. L'instrumentalité, comme caractère d'outil des objets (l'outil étant l'instrument devenu habituel ${ }^{2}$ ), est la source de significations et de rapports sans cesse nouveaux - et non l'analogie, comme le conclut Husserl. Antéprédicativement, les objets ont des fins déjà constituées dans le monde de la vie du fait de nos mouvements passés, ouvrant sur de nouvelles fins lorsque ces mêmes mouvements sont esquissés pour de nouveaux objets, mais aussi prédicativement, sur le plan symbolique, lorsque nous enseignons ou commandons aux autres de se servir des mêmes instruments, ou apprenons d'eux à le faire.

L'instrumentalité montre que la vie sensible n'a pas un accès direct, désintéressé au monde et aux choses; bien au contraire, la production médiatise la relation de la vie sensible aux choses. Autrement dit, la production est l'intentionnalité originaire, l'Urstiftung. Par conséquent, «le passage du sensible à l'intelligible ne peut se décrire correctement que par 1 'analyse des formes techniques et économiques de cette production ${ }^{3}$.

\footnotetext{
${ }^{1}$ Ibid., p. 409 [227].

${ }^{2}$ Ibid., p. 469 [316].

${ }^{3}$ Ibid., p. 405 [220]. Notons que John Dewey arrive à des conclusions très similaires. Voir "The Development of American Pragmatism » et "What I Believe », The Essential Dewey, vol. 1, Bloomington, Indiana University Press, 1998, p. 3-13, 22 -
} 28. 
Bref, le monde de la vie est le monde réel; l'ego transcendantal est l'être humain réel; les idées ont lieu et trouvent leur sens dans la vie sensible ; la phénoménologie apporte donc bel et bien « une genèse méthodique et positive de la conscience à partir de la vie ${ }^{1}{ }^{2}$, mais à corps défendant. Lorsqu'il est question du champ sensoriel, de la locomotion, de la préhension et de la manipulation, qui toutes donnent sa réalité à la chose, il est en fait question des structures du comportement en ce qu'elles donnent son sens au monde sensible. En prenant la phénoménologie à rebours de sa propre théorie, Thao peut donc passer de l'analyse du comportement à celle de la conscience, grâce à ce que la phénoménologie révèle, sans pourtant être à même d'accomplir :

Une étude scientifique de l'existence humaine, où l'explication par les conditions réelles ne supprime pas la compréhension des valeurs idéales, mais permet au contraire de les fonder et de les critiquer dans le sens même de leurs prétentions authentiques ${ }^{2}$.

Autrement dit, il s'agit de « comprendre le mouvement effectivement réel par lequel la nature devient humaine, en se constituant en esprit ». L'expérience matérielle est le fondement de l'expérience antéprédicative, et non le contraire.

C'est de la sorte que le matérialisme est à la fois la vérité et le contenu effectivement réel de la phénoménologie et de l'idéalisme transcendantal. Il doit à la phénoménologie l'abandon de l'attitude naïve (et l'on devine ici une critique voilée du matérialisme d'avant la phénoménologie), et élève la description du pur vécu vers une description de la constitution du monde par une subjectivité qui se trouve dans l'être comme nature qui prend conscience d'elle-même : cette part de la nature qui est plus précisément une part de l'animalité, qui est elle-même une part de la nature, mais toujours néanmoins nature, la subjectivité. Le sensible est bel et bien le fondement de la vérité mais le sensible est matériel et non simplement contenu d'expérience, il est fait, contenu d'expérience, vécu, sur le fond d'autres expériences et d'autres pratiques matérielles.

${ }^{1}$ Ibid., p. 403 [218].

${ }^{2}$ Ibid., p. 404 [218]. 


\section{L'intentionnalité repensée à partir et au-delà de Husserl}

Tran Duc Thao repense ainsi la conscience comme la vie qui devient autre chose qu'elle-même, tout comme la vie est la matière devenue autre chose qu'elle-même, de la même manière que le chimique "s'appuie" sur le physique $^{1}$. Les structures matérielles délimitent et définissent ce que peut être la vie, tout comme la vie offre ses structures à la conscience, bien qu'entre chaque mode d'être, le rapport demeure dialectique en ce que la conscience est en mesure d'agir sur la vie, et la vie sur le matériel. Thao, suggérant que les formes intentionnelles que décrit Husserl correspondent aux formes réelles que décrivent les recherches expérimentales, peut affirmer que « la conscience est le mouvement des conduites différées, ce qui nous renvoie aux circuits différés dans le trajet de l'influx nerveux ${ }^{2}{ }^{2}$, les centres supérieurs inhibant le fonctionnement autonome des centres inférieurs, mais s'appuyant néanmoins sur eux. De la sorte, c'est la réalité matérielle même qui est une dialectique, un mouvement de développement du contenu de chaque mode d'être du fait de ses structures en un mode nouveau, dans un devenir continu, dans une absorption qui est aussi maintien, suppression, conservation, dépassement, bref, Aufhebung par causalité interne. L'identité contient le changement.

C'est à partir de cette conception de la réalité matérielle que Thao conçoit la genèse de la conscience et est à même de repenser « la structure du sujet réel, à savoir le corps vivant comme centre de mouvements $»^{3}$. Les notions de conscience et de sujet conservent une grande part de leur signification philosophique traditionnelle, mais dans le contexte d'une plus forte matérialité. De la sorte, pour Thao, la nécessité est la réalisation de la liberté - c'est-à-dire que chaque mode d'être est formé et structuré comme une nouvelle nécessité, à partir d'une liberté propre au mode d'être sur lequel il s'appuie. La conscience se développe ainsi dans la liberté ouverte par les structures du comportement vital.

La question est alors de repenser l'intentionnalité en termes matérialistes. Thao définit l'acte de conscience par le comportement et la conscience par la dialectique qui est propre au comportement - elle en émerge donc. L'horizon de possibilités pratiques qui se dessine au contact d'un objet définit le sens vécu de cet objet comme ensemble d'esquisses de

\footnotetext{
${ }^{1}$ Thao reprend ici des idées présentées par Merleau-Ponty dans La Structure $d u$ comportement, Paris, PUF, 2013.

${ }^{2}$ Ibid., p. 427-428 [252].

${ }^{3}$ Ibid., p. 421 [243].
} 
comportement qui sont ensuite réprimées ou inhibées, ne laissant qu'un comportement qui pourra se déployer. Il n'y a aucune image de l'objet dans la conscience; l'extérieur devient plutôt intérieur par les comportements esquissés (noèmes) et réprimés (noèses) à son endroit. Autrement dit, la dialectique du comportement consiste en ce que la conscience de l'objet est le mouvement de répression d'esquisses de comportements, et que la conscience de soi est le maintien de ces esquisses en tant que supprimées. Ce maintien est la rétention des esquisses réprimées, de tout ce qui n'a pas été réalisé, dans une conscience de soi capable de réfléchir sur ce qui aurait pu être fait :

Les réactions réveillées par le stimulus et arrêtées par l'acte réel avant qu'elles n'aient pu arriver à la phase de l'accomplissement, s'intègrent dans le comportement total comme des moments supprimés, conservés, dépassés ${ }^{1}$.

La conscience est ainsi toujours à la fois conscience de l'objet et conscience de soi puisqu'elle est conscience des comportements esquissés en relation à l'objet. Pour le formuler autrement, la dialectique du comportement consiste en ce que le sujet se crée par la répression des comportements possibles, et toujours en relation et en réaction aux objets et aux comportements possibles qui s'esquissent autour d'eux, par leur appel.

Le comportement est la médiation entre le sujet et l'objet, ainsi qu'entre la conscience et elle-même - le corps et la vie demeurent matériels et permettent la subjectivité, elle-même objet. L'intentionnalité doit par conséquent être pensée en relation à un agir qui consiste à laisser faire, à ne pas réprimer un comportement appelé par un objet, voire par un autre comportement. Le sens intentionnel est limité aux actes qui s'esquissent, chacun ouvrant un autre horizon de comportements possibles. Par contraste, les actes réels, non réprimés, laissent une trace, qui devient une disposition à répéter une même structure générale de l'acte dans d'autres contextes bref, une habitude, issue du développement de ces dispositions en schèmes du comportement, qui à leur tour permettent de nouvelles esquisses qui définissent à leur tour, et autrement qu'auparavant, le sens intentionnel de l'objet. La conscience est l'objet qui devient sujet en produisant au sein de l'identité une différence, par le comportement. Elle est l'objet en vie qui revient et réfléchit sur ses propres comportements, s'ouvrant de la sorte à de nouveaux comportements. La prise de conscience, c'est-à-dire la réflexion, où la conscience coïncide avec son être objectif, est le remplacement par

${ }^{1}$ Ibid., p. 422 [244]. 
répression ou inhibition d'une forme ancienne du mouvement par une nouvelle forme, d'une habitude de comportement par une nouvelle action qui pourra aussi devenir habitude.

Agir - réaliser un comportement - et réprimer sont pour le sujet se porter vers son avenir immanent, c'est-à-dire vers l'horizon ouvert par la dialectique des esquisses et des habitudes. Thao précise que cet

avenir immanent « lui-même », comme sens constitué, est le même acte, mais en tant qu'il s'esquisse et apparaît dans son achèvement idéal comme cet avenir déjà présent qui se dessine dans l'horizon du champ ${ }^{1}$.

Thao voit donc l'avenir comme un horizon du champ sensoriel et du champ comportemental, déjà inscrit dans l'ensemble des esquisses réprimées et de l'esquisse achevée, et comme attaché au sens intentionnel des objets. L'avenir est immanent à la conscience en ce qu'il est attaché non pas à la conscience elle-même et à ses fiats, mais plutôt au comportement, qui est hors de la conscience ${ }^{2}$.

Pour comprendre cette formation du sens des objets, donc des habitudes, il est nécessaire de bien saisir ce que le comportement a d'universel, à savoir « la production, telle qu'elle émerge de l'usage de l'outil» ${ }^{3}$. L'universalité est donc matérielle, et non idéale - et il en va de même de la constitution du sens. L'usage répété de l'outil a pour conséquence la constitution de règles de l'activité productrice, c'est-à-dire la formation d'habitudes. Les mêmes conditions qui dictent l'usage de l'outil se reflètent dans la vie collective sur le plan symbolique qui s'articule sur la structure de la production - qui elle-même règle le comportement. Thao présente ici une théorie du reflet tout autre que celle du marxisme orthodoxe ${ }^{4}:$ il s'agit d'un reflet entre les plans individuel et collectif, et comportemental et symbolique. Thao réinterprète donc la dialectique de la base et de la superstructure chez Marx en des termes qui montrent bien la liberté d'action qui demeure au plan symbolique, et qui visent à trouver ce qu'il y a d'universel dans la production, plutôt que ce qui dépend de ses formes économiques et sociales.

L'importance de l'instrument permanent — l'outil — comme forme fixe de la production tient d'abord à ce que les comportements du corps

${ }^{1}$ Ibid., p. 435 [266-267].

${ }^{2}$ Ibid., p. 453 [293].

${ }^{3}$ Ibid., p. 452 [292].

${ }^{4} \mathrm{La}$ théorie marxiste du reflet, souvent associée à un marxisme «vulgaire », avait déjà été critiquée par Karl Korsch, dans Marxisme et philosophie, Paris, Éditions Allia, 2012, $144 \mathrm{p}$. 
propre, comme marcher, courir, s'asseoir, sont intégrés à la production et donc transformés au contact répété de l'outil. De la sorte, le sens de ces comportements et la manière dont ils se déploient dépendent de l'objet de la production accomplie par cet outil. Ensuite, cette importance tient à ce que des techniques - terme par lequel Thao entend simplement des habitudes collectives - émergent de la structure matérielle des outils, structure qui est un horizon d'usages possibles, comme une structure intrinsèque de la production qui peut être saisie sous forme de règles. Autrement dit, il n'y a pas de comportement sans production (et il y a toujours production); l'usage collectif des outils, leur passage d'une main à l'autre, l'apprentissage qui va avec leur maniement, forment une série de règles qui se répètent chez tous ceux qui participent à cette même production, réglant leur comportement, non seulement dans l'horizon de la production, mais dans l'ensemble du comportement. Les habitudes acquises de la sorte sont bel et bien collectives, puisqu'une même structure existe du fait d'un comportement commun, collectif. Le plan comportemental se trouve donc à la fois sur le plan individuel et sur le plan collectif, porté par la relation dialectique entre ces deux plans.

Le langage émerge de l'activité productive : le sujet parle à l'autre pour apprendre de lui ou lui apprendre comment manier un instrument; le sujet apprend sa pensée en la disant, et ne dit, ne parle, qu'en arrêtant de travailler, pour parler du travail, pour parler de ce qu'il fait. Le symbolique reflète donc le comportemental et permet de préciser le sens intentionnel des objets et surtout des outils : il est retour sur le comportement, afin de le continuer - ce qu'est d'ailleurs la conscience, comme retour sur les esquisses réprimées et mises en acte. Nous pouvons ainsi voir le sens que prend la réflexivité : «L'universalité n'est qu'un résultat où se réfléchit la possibilité indéfinie de répétition impliquée dans la structure objective du processus de l'outil ${ }^{1}$.» Un concept est universel et est en accord avec les choses parce qu'il émerge de l'activité productrice sur les choses, parce que la réflexion est retour sur le comportement, lui-même réglé autour de la production. Et si la production oriente tout le comportement, c'est aussi qu'originairement le langage dans son exercice «opère une transposition imaginaire des schèmes producteurs élémentaires sur toutes les données perceptives $»^{2}$. Après tout, les concepts servent à mieux s'emparer et se saisir des choses, qui seront alors perçues autrement, selon un horizon de sens transformé par le langage. Toute chose peut ensuite entraîner des esquisses

${ }^{1}$ Ibid., p. 453 [293].

${ }^{2}$ Ibid., p. 454 [294]. 
d'un comportement productif. Ainsi, le plan symbolique se trouve présent à la fois sur le plan individuel et sur le plan collectif.

La conscience est un mouvement matériel qui permet l'accomplissement du comportement : « Il n'y a rien de plus dans le mouvement intentionnel qu'un mouvement réel esquissé et réprimé ${ }^{1}$.» L'action est prolongée et soutenue par le vécu, par la conscience qui répète les esquisses et dirige ainsi l'action en maîtrisant les niveaux antérieurs du comportement, comme la perception, la locomotion, et la manipulation, donc en inhibant ou en réprimant des esquisses de perceptions et de mouvements - sans jamais pouvoir se diriger elle-même de la même manière. La conscience permet certes le comportement symbolique, où elle joue un rôle plus direct, mais l'expression ne demeure que l' " achèvement symbolique de l'acte ébauché »" ou que son achèvement ébauché - l'achèvement comme tel demeure toujours à faire. Le reflet qui constitue la conscience est ainsi un renversement : la conscience émerge du comportement, puis semble le contrôler effectivement, mais ne fait que permettre une anticipation.

\section{Conscience matérielle, conscience pure, et collectivité}

Suite à ces développements, nous pouvons saisir la genèse de la conscience dans la création toujours originaire qu'est la production, qui est la manière dont elle agit son ancrage dans l'être. Il nous reste à terminer la description de cette genèse (dans l'intentionnalité), à la mettre en relation avec l'origine (dans l'histoire individuelle et collective) de la conscience, et à voir comment Thao explique l'origine de l'idée de conscience pure.

Le travail, comme tout comportement, est extérieur à la conscience. Il est un mouvement par lequel «le donné s'absorbe dans le produit, et se pose comme tel pour la conscience dans l'acte de jugement $»^{3}$. Autrement dit, le sujet se retrouve dans les choses par la production et en transformant le donné en produit, trouve un premier degré de liberté, fort limité, et seulement par rapport aux choses. Ce même mouvement comportemental ouvre par ailleurs une structure des rapports humains, que la conscience ne peut saisir ni maîtriser. Les relations sociales ont ainsi lieu sur le plan collectif, qui se trouve être avant tout comportemental, plutôt que d'être entièrement symbolique.

${ }^{1}$ Ibid., p. 456 [297-298].

${ }^{2}$ Ibid., p. 456 [299].

${ }^{3}$ Ibid., p. 457 [300]. 
Du point de vue de la production collective, la grande diversité et le manque d'intégration des entreprises productives ouvrent la nécessité de l'intégration de «la totalité des producteurs dans l'unité d'une travail commun $\rangle^{1}$ et pointe déjà vers la collaboration en vue de la production et les solidarités tissées grâce à l'échange, à un niveau localisé. Toutefois, cette même diversité implique aussi différences et séparations entre les travaux, empiétement de ces travaux et des objectifs les uns sur les autres et donc entraves, ainsi que convoitises. De cette face conflictuelle de la diversité des travaux et des activités productives - donc des schèmes du comportement et des échanges symboliques - émerge la logique de l'accaparement. Cette logique limite le processus général de la production, même s'il peut profiter à quelques-uns.

La force de la logique de l'accaparement, du fait des dangers associés au conflit, fait qu'elle l'emporte sur la logique de la collaboration, qui quant à elle est intégrée et redirigée en vue de l'accaparement. Dans cette logique, tout échange apparait comme un rapport de droit, transcendant les nécessités économiques. Les conséquences de la concentration sur l'effort d'accaparement sont le déni du réel, le déni de la coopération, le déni de l'équivalence du donné qui est transformé par la production, le déni de la co-participation à la production et donc le déni de la propriété commune du donné, et enfin l'exclusivité de la propriété.

L'idée de conscience pure, constituée idéalement et à l'exclusion des autres, émerge de cette logique de l'accaparement et surtout de la propriété exclusive :

Avec le passage aux rapports économiques de production ou rapports d'échange, la forme de la possession aboutit à enfermer chacun dans les limites de son développement propre et à le constituer idéalement en une pure conscience de soi négatrice de toute extériorité comme telle, puisque la réalité elle-même n'est désormais admise que dans l'horizon d'une possession possible ${ }^{2}$.

Autrement dit, ce n'est plus seulement la production qui structure le comportement, mais aussi la forme des échanges et la possession des produits de la production. Et la conscience qui émerge de ces échanges, qui tente de les continuer et les rend possible en réprimant les autres esquisses - extrêmement complexes à ce stage — de comportements (comme les comportements

${ }^{1}$ Ibid., p. 457 [300].

${ }^{2}$ Ibid., p. 459 [302]. 
collaboratifs ou de don) se constitue par réflexion dans le même mouvement de séparation et d'exclusion qui caractérise ces échanges.

Le sujet ne voit alors plus l'objet que par son projet d'accaparement : la conscience réprime et inhibe toute autre esquisse, liée à tout autre projet, et ne voit plus que les conditions réelles qui décident de la manière dont il peut être possédé. Bref, la conscience en vient à nier l'objectivité réelle, à nier l'extériorité ; elle se fait entièrement sujet et perd de vue sa propre objectivité. La propriété privée, qui revient à l'expropriation de tous les autres de la propriété, est la création d'une transcendance: le sujet ne voit l'objet, l'extériorité, que comme extérieur à lui-même, sans aucun lien avec lui, et donc simplement à posséder parce que libre de toute attache à tout sujet. La position de transcendance est ainsi créée par la conscience qui se sépare de ses attaches au matériel afin de le séparer de ses attaches aux autres consciences, et afin de pouvoir ainsi l'échanger, l'acquérir. Ajoutons que tout comme Husserl ne peut revenir au monde de la vie qu'il a quitté, la conscience ne peut plus voir l'objet que comme une image en elle-même ; que tout objet, même le corps propre, peut devenir une propriété. La conscience ne se voit alors que comme propriétaire, transcendant la réalité de ce qu'elle possède, dans une "appropriation idéale du monde » ${ }^{1}$. Se voir comme pure spiritualité, pure conscience de soi, c'est refléter symboliquement le comportement d'échange et oublier jusqu'à la production, qui ellemême devient une affaire d'échange (de temps ou de force de travail, contre rémunération).

Thao définit donc la transcendance comme la prétention de la conscience à posséder la totalité de l'être, et à ne permettre à autrui de posséder quoi que ce soit seulement parce que la conscience y consent. Ce «mouvement réel des concessions réciproques $»^{2}$ est transposé sur le plan idéal en état de droit, où tout repose sur le consentement de la conscience, sur la conscience même ; dans l'état de droit, la conscience est l'origine de tout. De la sorte, «le sujet s'exalte dans la certitude intime d'une liberté absolue, où chacun se retrouve dans l'être-autre en le constituant en soi-même $»^{3}$. Tout le projet d'une phénoménologie constitutive se trouve ainsi récusé, puisqu'il réduit la conscience à sa structuration accaparatrice.

${ }^{1}$ Ibid., p. 459 [303]. Ici Thao cite en longueur le passage sur la base et la superstructure de la préface de la Contribution à la critique de l'économie politique de Marx.

${ }^{2}$ Ibid., p. 466 [312].

${ }^{3}$ Ibid., p. 466 [312]. 
La constitution d'une pure conscience de soi qui se détache symboliquement de la matérialité sans pour autant pouvoir s'en détacher dans son comportement n'est pas la seule conséquence de la propriété exclusive tout un système de droit est érigé sur les mêmes bases, et sur la même fiction individualiste. L'expropriation des autres producteurs dépend d'une conception de la propriété qui

ne consiste pas dans la simple jouissance de l'objet, mais bien dans l'exclusion de droit de toute participation d'autrui, alors qu'il appartient précisément à l'être réel de la valeur d'usage de pouvoir servir à tous ${ }^{1}$.

Ainsi, la reconnaissance de la propriété d'autrui est l'une des esquisses réprimées par les habitudes de la conscience pure, accaparatrice et possessive. Le résultat en est l'habitude de consentir à la propriété des autres, de consentir au partage, de consentir aux échanges, comme s'ils ne dépendaient que de la conscience, comme s'il pouvait y avoir même consentement.

Alors que Husserl tente de rejoindre autrui à partir de la sympathie pour autrui et de la projection en autrui, et par là d'établir les bases de l'intersubjectivité, et tout comme ce consentement ne reconnaît jamais autrui comme semblable, c'est l'esquisse qui permet à Thao de comprendre autrui. La reconnaissance de la propriété d'autrui est un comportement esquissé par la conscience puis réprimé - ce qu'elle réprime la constitue encore plus que ce qu'elle achève, rappelons-le —, esquisse qui démontre qu'autrui nous appelle, nous interpelle à agir, à collaborer et à le respecter, et s'ouvre à la fois à notre accaparement et à notre exclusion. Or, tout comme la production est liée à des techniques qui règlent le comportement et ainsi la conscience qui le continue, le droit codifie des comportements et règle aussi la conscience, favorisant certains échanges et menant à la répression des esquisses de comportements collaboratifs et co-participatifs.

Thao explique de la sorte ce que présupposait Husserl : la conscience individuelle pure. L'idée de conscience pure vient du privilège donné à la conscience, à l'encontre du comportement, privilège qui lui-même vient d'une conception exclusive de la propriété, qui est permise par les conditions matérielles de sa production. Elle vient aussi de la position des idéalités, des essences et de la philosophie pure non pas dans «les couches primitives et proprement animales $»^{2}$ de la vie humaine, mais au contraire dans les significations humaines propres à l'expérience de la conscience de soi. Or,

${ }^{1}$ Ibid., p. 462 [304].
${ }^{2}$ Ibid., p. 456 [297]. 
les sociétés et les formes de la production sont trop complexes pour permettre la connaissance de soi claire que suppose l'idéalisme, surtout lorsque le soi est coupé de ses assises comportementales.

Le matérialisme dialectique permet toutefois de surmonter les difficultés de la fiction de la conscience pure. Après tout, sous ce contenu négatif et tous ces dénis, il demeure un contenu positif «qui n'en fonde pas moins la signification authentique de l'existence spirituelle ${ }^{1}$ et que le matérialisme dialectique est à même de dégager. Thao se propose donc de décrire ce qu'est l'existence spirituelle, si ce n'est pas la conscience pure, et de trouver l'universalité réelle dans la joie mystique et mystifiante d'un soi universel à l'origine de tout et consentant à tout. Cette véritable universalité, cette vérité de l'existence, trouve elle aussi ses origines dans le travail. Non pas le travail individuel, qui n'est que mystification, mais le travail social, c'est-à-dire le travail dans ce qu'il a toujours de social et que la conscience accaparatrice réprime. Nous l'avons déjà vu, en revenant et en réfléchissant sur le travail, la conscience élabore des concepts pour augmenter sa prise sur les choses et sur son activité et prolonge la production par des esquisses symboliques de la tâche dans sa totalité. Ce prolongement inclut ce que la tâche a de commun et ce en quoi elle nécessite la participation de tous, et donne à la production son caractère universel. Or, la répression des actes d'accaparement esquissés dans d'autres formes d'échanges, coupés de la totalité, permet à cette conscience de s'unir aux autres, et par là, de se réaliser : cet échange dans le travail commun s'impose parce qu'il permet d'utiliser à leur meilleur escient les forces de production. Les mots, les concepts qui émergent de ces tâches et de ces comportements accomplissent «l'unité du soi et de l'autre $»^{2}$ dans une existence commune, dans « la totalité sociale $»^{3}$. La conscience se réalise parce qu'à partir de l'immanence, elle réussit à se dépasser vers une existence commune par une tâche transcendante, où le tout, c'est-à-dire la société, peut réaliser ses besoins. Chacune y gagne, parce que cette transcendance matérielle élimine la source des exclusions réciproques et de l'infini des désirs.

\footnotetext{
${ }^{1}$ Ibid., p. 466 [312].

${ }^{2}$ Ibid., p. 466 [313].

${ }^{3}$ Ibid., p. 467 [314].
} 


\section{Conclusion : L'universalité à réaliser}

L'universalité est donc pour Thao le dépassement de soi par la coopération, suivant les exigences de la production, elles-mêmes suivant les exigences de la vie et de la liberté qui s'y esquisse. Un tel travail est le « sens proprement humain ${ }^{1}{ }^{1}$ de la vie humaine. L'universalité est d'ailleurs déjà esquissée au sein de la production propre au prolétariat - qui intervient fort tard dans cette analyse (analyse qui ne se concentre donc pas sur le capitalisme) : la «suppression de la forme privative de l'appropriation elle-même » ${ }^{2}$ sera aussi celle des égoïsmes particuliers alors que le prolétariat reprendra possession «au profit de l'humanité productrice des significations humaines [s'entend, matérielles] détournées de leur sens humain par la coalition spiritualiste des intérêts privés ${ }^{3}$. L'universalité réelle - matérielle et non mystifiante - n'est possible que dans la société communiste, qui abolit les intérêts privés, qui inhibe la logique de l'accaparement, qui libère ainsi l'action humaine vers un avenir qui est déjà immanent à la totalité sociale, mais sans cesse réprimé, et qui libère ainsi les consciences d'habitudes, de techniques, et de symboles qui empêche ces consciences de se réaliser.

De la sorte, en retraçant l'origine et la genèse de la conscience pure et la manière dont cette conscience pure occulte le matériel et donc une partie d'elle-même et en retraçant l'origine et la genèse de la conscience dans le travail, Tran Duc Thao montre les origines possibles d'une conscience matérielle qui soit aussi conscience de sa matérialité, et qui pourrait se vivre comme commune. Il cherche à rendre possible la formation d'une conscience qui est celle d'individus qui participent à la totalité sociale et où les plans comportemental et symbolique de leur existence arrivent à favoriser cette participation et, par là, l'universalité, la vérité, l'honneur, la charité, le devoir, la justice et la liberté. L'horizon révolutionnaire du marxisme semble lui donner une direction vers une telle conscience et une telle société, mais comme le montreront les travaux ultérieurs de Thao, ce passage demeurera tout entier à accomplir... en pratique, afin de transformer le travail et la production. Ce pourquoi, peut-être, Thao quitta la France pour se joindre à l'effort révolutionnaire au Vietnam, dirigé contre la France et son économie accaparatrice, mais aussi ouvrant cet horizon révolutionnaire.

Nous avons tenté ici de montrer comment Thao continue la tradition phénoménologique, la reprenant tout en brisant avec elle pour la faire sienne,

\footnotetext{
${ }^{1}$ Ibid., p. 497 [363].

${ }^{2}$ Ibid., p. 498 [364].

${ }^{3}$ Ibid., p. 470 [318].
} 
et la combinant avec un matérialisme dialectique qui lui est propre ${ }^{1}$. Un travail autour des hypothèses qu'il s'agit d'une phénoménologie non husserlienne, c'est-à-dire reprenant l'impulsion donnée par Husserl, mais passant par le domaine de la production plutôt que de la signification, ainsi que d'un matérialisme dialectique non marxiste nécessiterait une étude plus rapprochée et du communisme vietnamien et du communisme français de l'époque, autant que du rapport de Thao à la phénoménologie. Néanmoins, ces mêmes emprunts à la phénoménologie et l'accent que Thao met par ailleurs dans Phénoménologie et matérialisme dialectique sur le comportement animal et l'acquisition du langage chez l'enfant - analyses que nous ne pouvons reprendre ici, et que Thao corrigera par ailleurs souvent dans les décennies qui suivront son retour au Vietnam — placent déjà son matérialisme en opposition à celui des marxistes de l'époque.

Dans cette direction, marquons toutefois que des parallèles importants avec Merleau-Ponty, qui demeureront ici inexplorés pour des raisons d'espace, apparaîtront au lecteur qui est aussi familier avec les textes de ce philosophe qui fut proche de Thao pendant plusieurs années : l'accent mis sur le comportement répétant et entraînant ailleurs plusieurs des analyses de La structure du comportement ${ }^{2}$; l'étude de l'acquisition du langage qui est contemporaine de celle des cours à la Sorbonne sur la psychologie de l'enfant ${ }^{3}$; l'étude du comportement animal qui précède celle des cours sur la nature $^{4}$; la recherche d'une philosophie dialectique au-delà de celles de Hegel et $\mathrm{Marx}^{5}$; le vocabulaire de la répression et de l'inhibition qui font écho à la formule que agir, c'est laisser faire ${ }^{6}$; ou encore la critique du

\footnotetext{
${ }^{1}$ Matérialisme dialectique qui peut, avec Sartre et A. Feron, être nommé un « marxisme ouvert ». Alexandre Feron, «Le marxisme ouvert de Trần Đức Thảo », La revue du projet, 51 (nov. 2015). En ligne : http://projet.pcf.fr/79166.

${ }^{2}$ M. Merleau-Ponty, La structure du comportement, Paris, PUF, 2013.

${ }^{3}$ M. Merleau-Ponty, Psychologie et pédagogie de l'enfant. Cours de Sorbonne 19491952, Lagrasse, Verdier, 2001.

${ }^{4}$ Maurice Merleau-Ponty, La Nature. Notes de cours du Collège de France, Paris, Seuil, 1995.

${ }^{5}$ Voir les manuscrits des cours de 1955-1956 «La philosophie dialectique » et « Textes et commentaires sur la dialectique », dont les notes toujours inédites ont été déposées à la Bibliothèque nationale de France. Voir sur ce point D. Belot, « Dialectique, ontologie et histoire dans les notes préparatoires aux cours sur La philosophie dialectique (1956) », Revue internationale de philosophie, 244 (2008), p. 189-206.

${ }^{6}$ Voir notamment M. Merleau-Ponty, «L'ontologie cartésienne et l'ontologie d'aujourd'hui », Notes de cours 1959-1961, Paris, Gallimard, 1996.
} 
marxisme orthodoxe à travers la notion d'institution (Stiftung) ${ }^{1}$. De tels rapprochements marquent par-dessus tout la dette de Tran Duc Thao à l'égard de Merleau-Ponty, leur fréquentation des mêmes inédits de Husserl, mais aussi leur projet partagé (mais non mené en commun) de dépasser et la phénoménologie husserlienne et le matérialisme dialectique marxiste.

\footnotetext{
${ }^{1}$ M. Merleau-Ponty, Les aventures de la dialectique, Paris, Gallimard, 2000 [1955] ; L'institution. La passivité. Notes de cours au Collège de France (1954-1955), Paris, Belin, 2003.
} 